\title{
Analysis of observed contamination through SAGE III's first year on orbit
}

\author{
Michael P. Thompson ${ }^{\mathrm{a}}$, Joshua Bangert ${ }^{\mathrm{a}}$, Samual Porter ${ }^{\mathrm{a}}$, David Flittner ${ }^{\mathrm{b}}$, Kaitlin Liles $^{\mathrm{b}}$, \\ Ryan Stanley $^{\mathrm{a}}$, and Elaine Seasly ${ }^{\mathrm{b}}$ \\ ${ }^{a}$ Science Systems and Applications, Inc., 1 Enterprise Pkwy, Hampton, VA, USA 23666

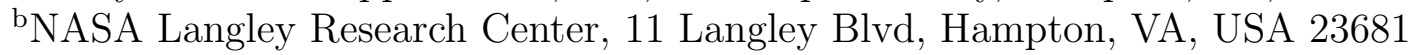

\begin{abstract}
SAGE III is a payload on the International Space Station that conducts measurements of ozone and other atmospheric constituents through the use of a moderate resolution spectrometer with an operating wavelength range of $290 \mathrm{~nm}$ to $1550 \mathrm{~nm}$. Because of the optically sensitive nature of the payload, a suite of eight Thermoelectric Quartz Crystal Microbalances(TQCMs) were included to monitor the operating environment. During the first year of operation, the SAGE III TQCMs were instrumental in detecting several periods of higher contamination and localizing their sources. A clear window made from quartz crystal covers the instrument assembly's aperture. Under nominal operating conditions, this window is only open during science gathering activities. However, if the rates of contamination accumulation are detected to be above the background rate, the window will be kept closed during science gathering to protect the optically sensitive instrument mirror. An analysis of the signal transmissions through the window for the wavelengths of $290 \mathrm{~nm}$ to $1550 \mathrm{~nm}$ has been conducted to determine any possible degradation of the window and potential influence on science data collected to date, and established a baseline for future analysis.
\end{abstract}

Keywords: SAGE III, Thermoelectric Quartz Crystal Microbalance, Optical Transmission, Dragon, Soyuz, Progress, International Space Station

\section{INTRODUCTION}

The SAGE III instrument is a moderate resolution spectrometer capable of capturing spectra in the wavelength range or $290 \mathrm{~nm}$ to $1550 \mathrm{~nm}^{1,2}$ SAGE IIIs objective is to measure concentrations of ozone, aerosols, water vapor, nitrogen dioxide, and other trace gases. Due to the nature of the instrument and the desire to acquire science data ,especially at the lower wavelengths for as long as possible, the effect of contamination on the payload was of high concern. As a result a suite of eight thermoelectric quartz crystal microbalances(TQCMS) was included on the payload. These TQCMs were divided into two sensor arrays called contamination monitoring packages (CMP). CMP 1 consists of five sensors one each pointing in Ram, Starboard, Nadir directions, and two ,one with a reduced field of view, pointing in the direction of Node 2. Figure 1 shows a layout of the sensor locations on the payload. This architecture provides $360^{\circ}$ coverage in azimuth and nadir. The TQCMs used were QCM research Mark 24 models with $15 \mathrm{MHz}$ crystals and a mass sensitivity of $1.965 \times 10^{-9} \frac{\mathrm{g}}{\mathrm{Hz*cm}^{2}} .^{3}$

The TQCMs output the difference in resonant frequencies between one crystal that is kept clean and one who's gold coated surface is exposed to the environment this is called the beat frequency. The beat frequency is linearly correlated to the mass adsorbed on to the sensor through the sensitivity coefficient. SAGE IIIs TQCM beat frequency is sampled at $1 \mathrm{~Hz}$. The data here is one minute averages of this data. On orbit TQCMs are known to be susceptible to the effects of incident solar flux. ${ }^{4}$ This is visible in the SAGE III TQCM beat frequency as an orbital variation in the beat frequency. In order to account for this any adsorption totals were calculated by using the difference between orbital maximum frequencies. Each TQCM has its temperature controlled via a thermoelectic cooler (TEC) with an operation range of $-20^{\circ} \mathrm{C}$ to $80^{\circ} \mathrm{C}$. Through the first seven months on

Further author information: (Send correspondence to Michael P. Thompson)

Michael P. Thompson: E-mail: michael.thompson@nasa.gov, Telephone: 757-864-7383 

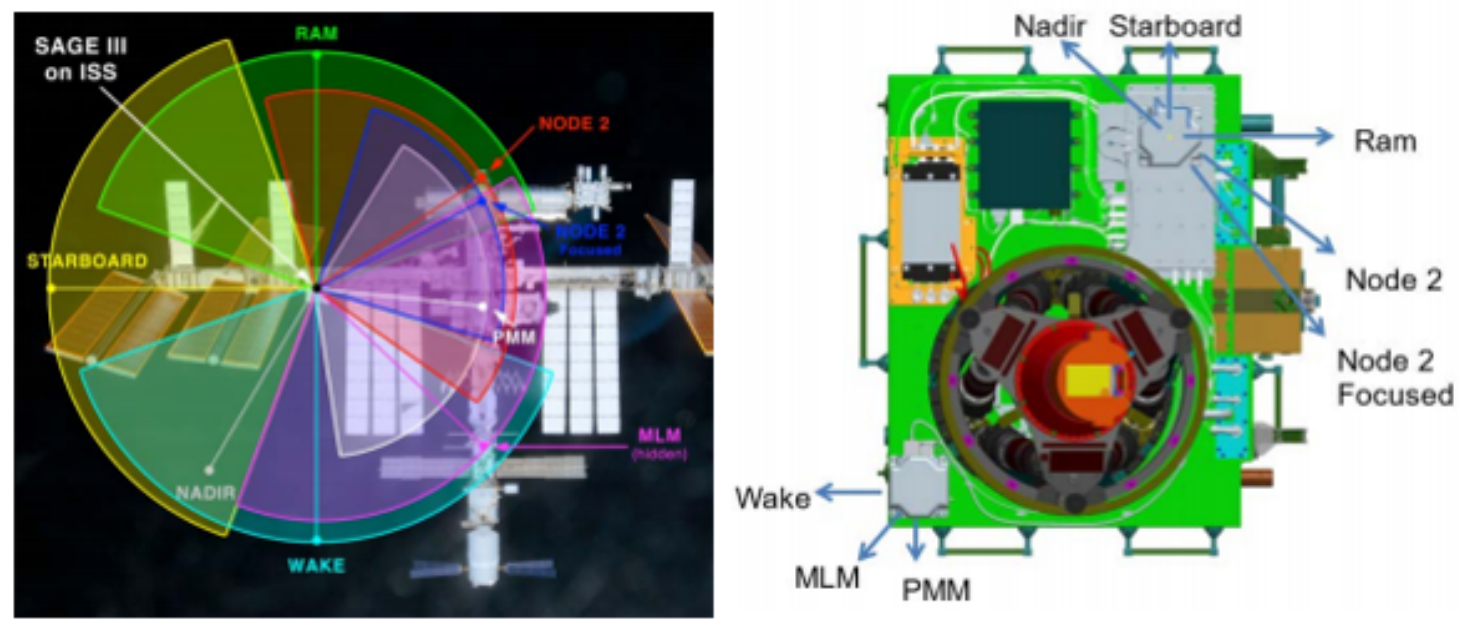

Figure 1. SAGE III TQCM pointing configuration

orbit all eight TECs were set to $-10^{\circ} \mathrm{C}$. In October 2017 in order to better understand the amount of adsorption onto the SAGE III sensitive surface the TEC for the Node 2 Focused TQCM was changed to $24.9^{\circ} \mathrm{C}$ which is an approximate average of the temperatures of the sensitive surfaces based on their correlation to the nearest temperature sensors.

\section{CONTAMINATION OBSERVATIONS}

When SAGE III first reached orbit the CMPs and their respective TQCMs were used to asses the contamination environment surrounding the payload. Once it was determined that environment was benign the contamination window was enabled for nominal operations and began opening before each science event. The team continued to monitor the TQCMS for unexpected or large increases in the beat frequency. These observations were crucial to alerting the SAGE III team to the elevated rate of contamination during the berthing of CRS-11's Dragon capsule in June 2017. As well as characterizing the contamination signatures of visiting vehicles

The starboard facing TQCM faces outboard along the truss of station. There is little station structure in this direction the largest portion of which is solar panels. This direction was chosen as the stow direction for our instrument as it provided the best compromise between thermal constraints and having our sensitive surfaces face a direction with the least potential for contamination. Figure 2 the beat frequency of the starboard TQCM has displayed an small but steady increase in adsorbed material over the first year on orbit

The ram TQCM faces the direction that approximately half of our science events occur in. Figure 3 is the beat frequency for this TQCM. Through the first 2 months on orbit material desorbed from the. Beginning in May 2017 material began to adsorb onto the sensor. There were three notable large contamination event observed by this sensor. These were the visits of SpaceX CRS vehicles 11,12 and 13. There is an obvious correlation between the beta angle and the amount of material adsorbed onto the sensors during each visit.

The Node 2 facing TQCMs field of view contains a large amount of stations structure. There are two of these TQCMs the first has a $70^{\circ}$ half angle field of view centered on node 2 , the second is also centered on node 2 wish a $50^{\circ}$ half angle field of view. These sensors have adsorbed the most material while on orbit due to completely encompassing the dragon capsules of CRS-11,12, and 13. Figure4 is the beat frequency of the node 2 focused tqcm. This TQCM has been noisy since SAGE III reached orbit. When the noise was not present the material adsorption is less than the node 2 sensor but is proportional to that TQCM. Figure 5 is the beat frequency of the node 2 facing sensor. This sensor has adsorbed the most material while on orbit. Both TQCMs have observed three periods of elevated absorption rates that correlate to the visits of CRS-11,12, and 13 . CRS related absorption remains on the TQCMs despite multiple attempts to burn off the material by raising the TEC setpoint to $80^{\circ} \mathrm{C}$. The amount of material absorbed during each CRS visit had a positive correlation to 


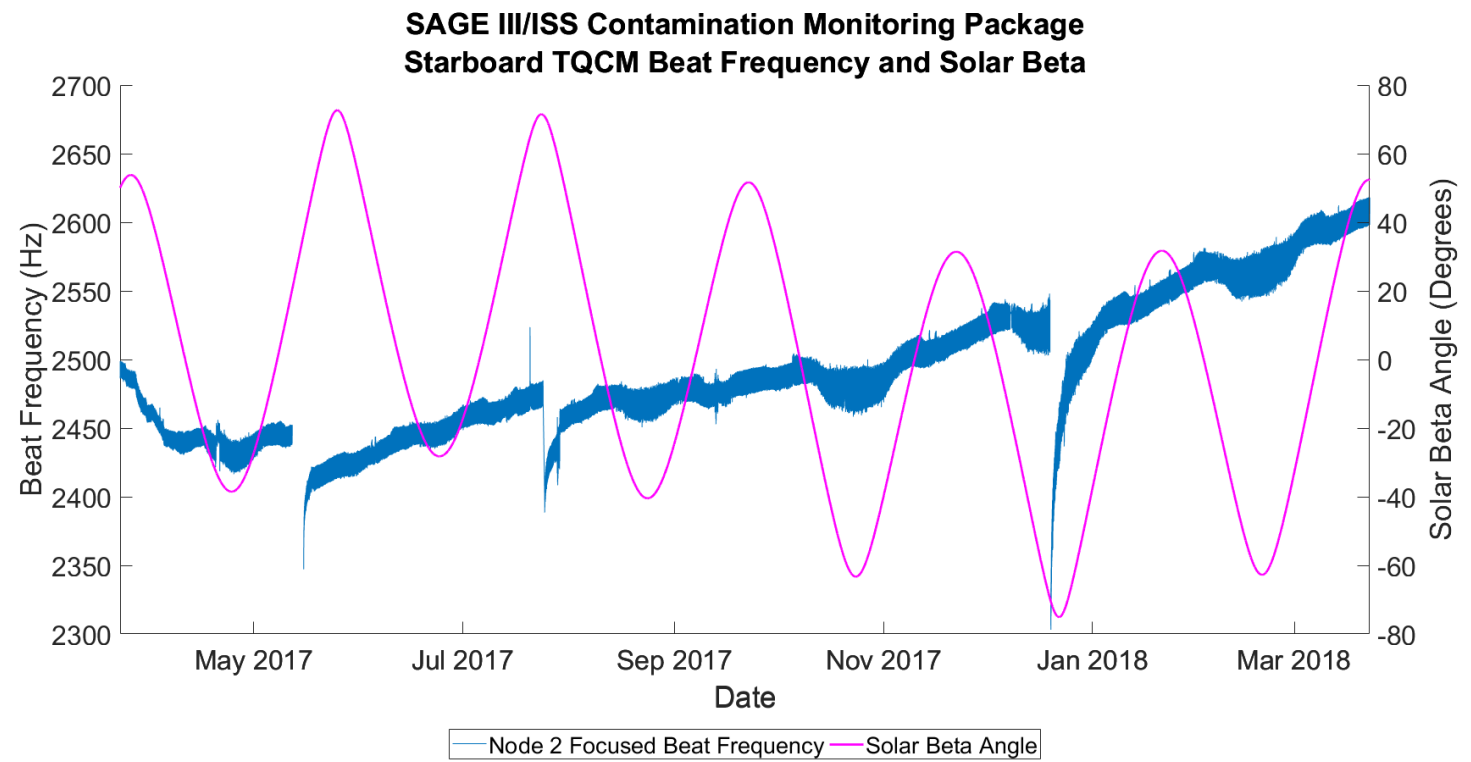

Figure 2. SAGE III Starboard facing TQCM beat frequency

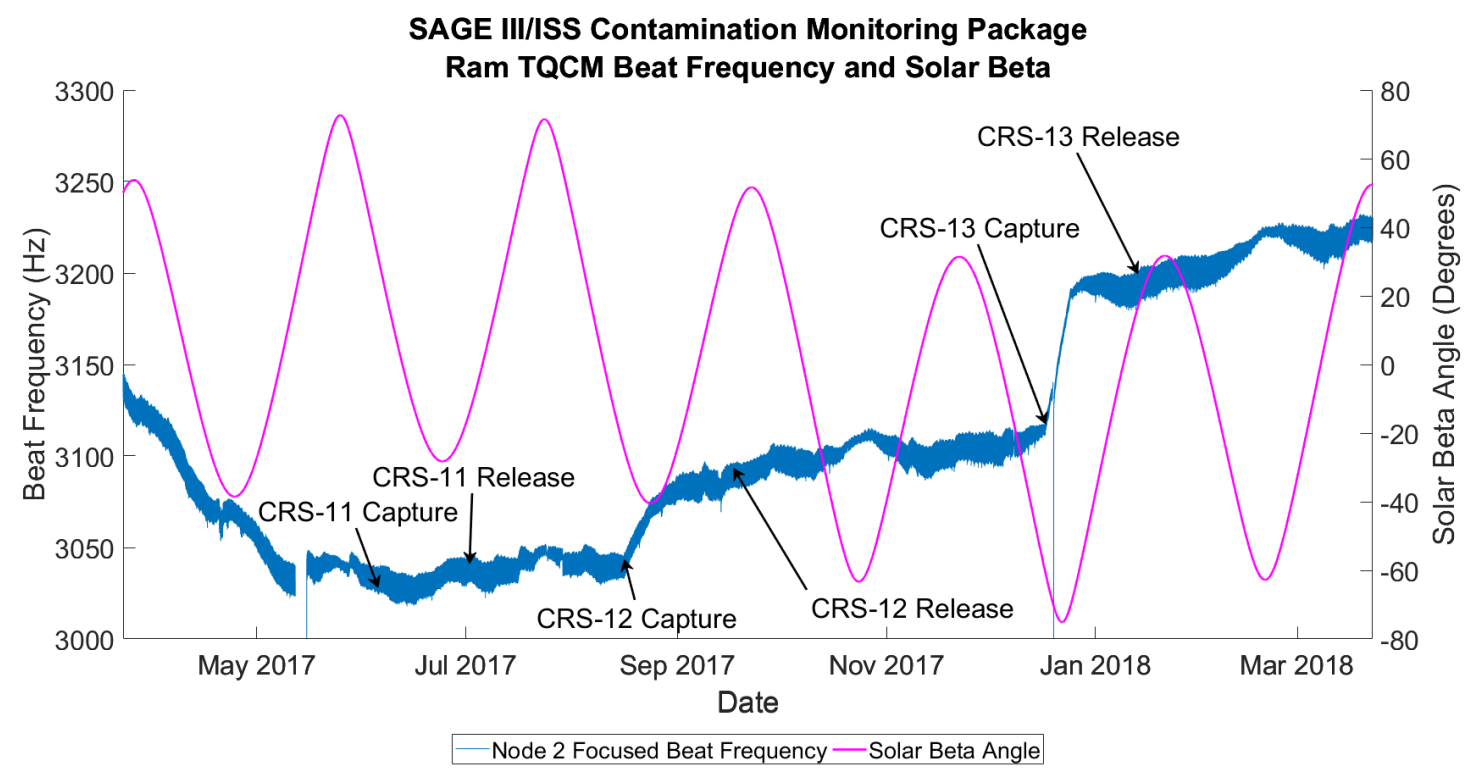

Figure 3. SAGE III Ram facing TQCM beat frequency

how negative the solar beta angle. The more negative the solar beta angle the more material absorbed by the TQCMs

The nadir facing TQCM has a field of view of empty space. It occasionally will adsorb material coming off of approaching vehicles. Figure 6 shows the beat frequency of this TQCM. Through one year on orbit this is the on of two TQCMs a net desorption of material during the first year on orbit.

The wake facing TQCM has a field of view of the rear of station and is the direction that the other half of 


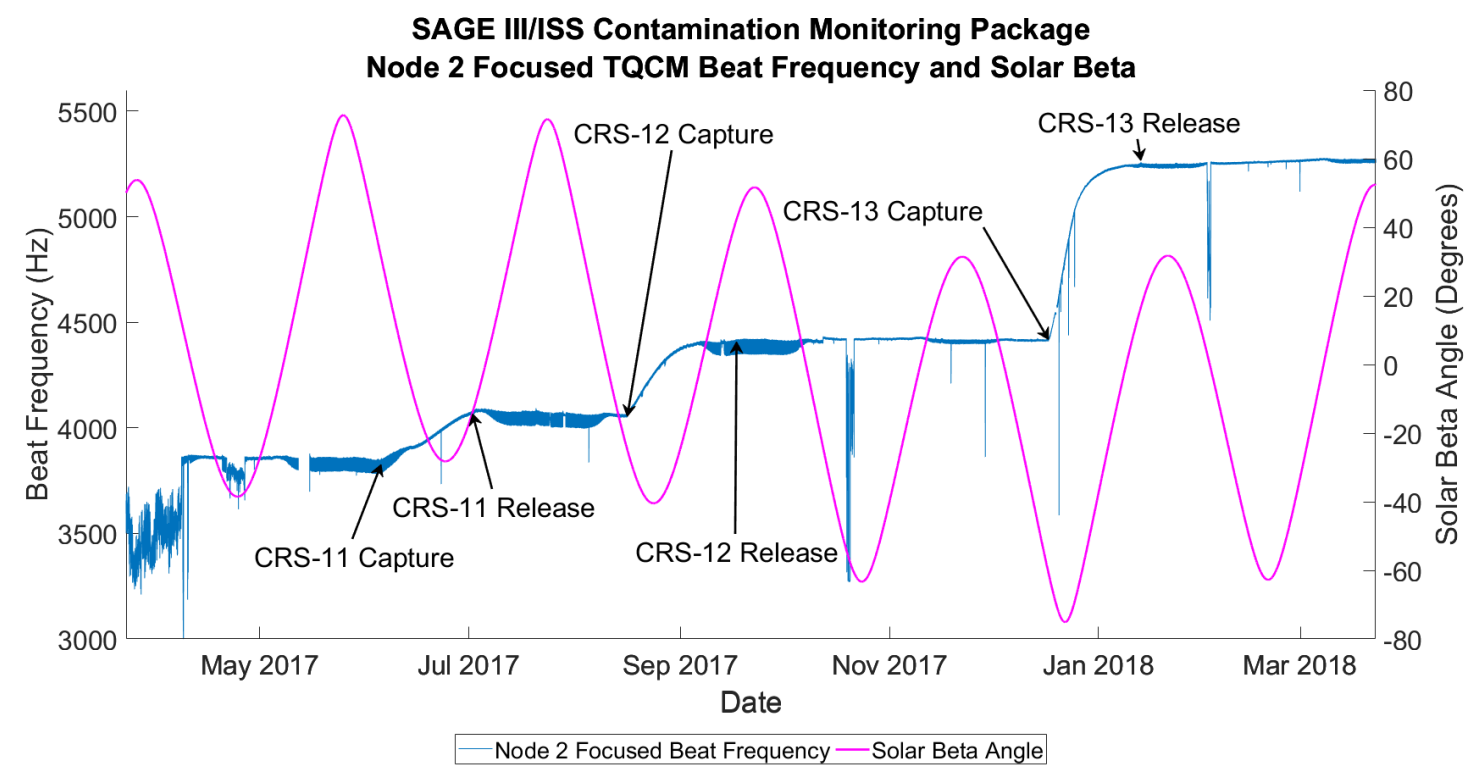

Figure 4. SAGE III Node 2 facing focused TQCM beat frequency

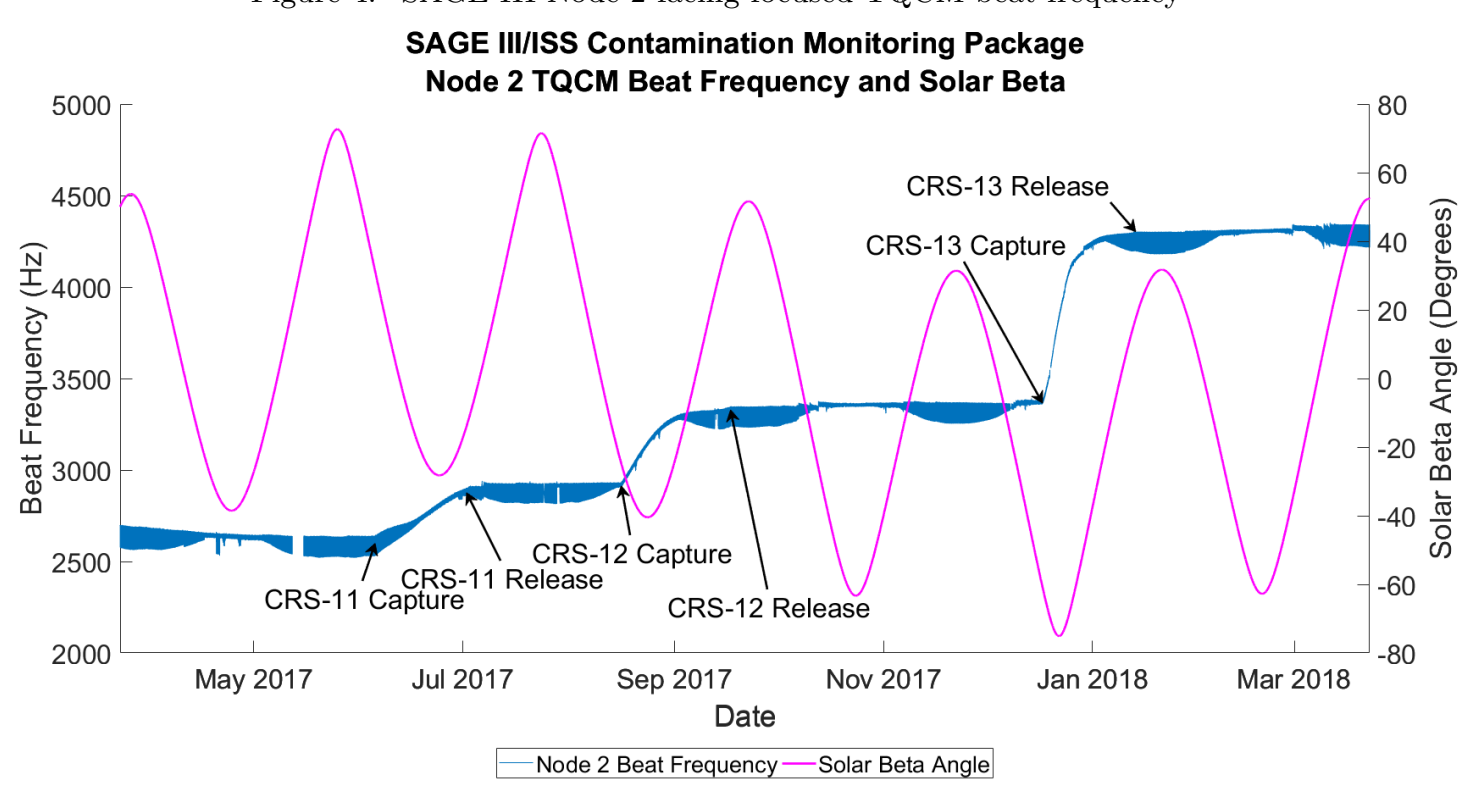

Figure 5. SAGE III Node 2 facing TQCM beat frequency

SAGE III science events occur in. The field of view includes several docking locations for Soyuz and Progress vehicles. Figure 7 shows the beat frequency of the wake TQCM. This sensors beat frequency displays several temporary increase that correlate to the arrival of either Soyuz of Progress vehicles to station. The amount of adsorption is correlated with the more negative beta angles after a period of time the material associated with these vehicles always desorbs from the TQCM. This TQCM also displayed a net desorbtion of material during SAGE IIIs first year on orbit 


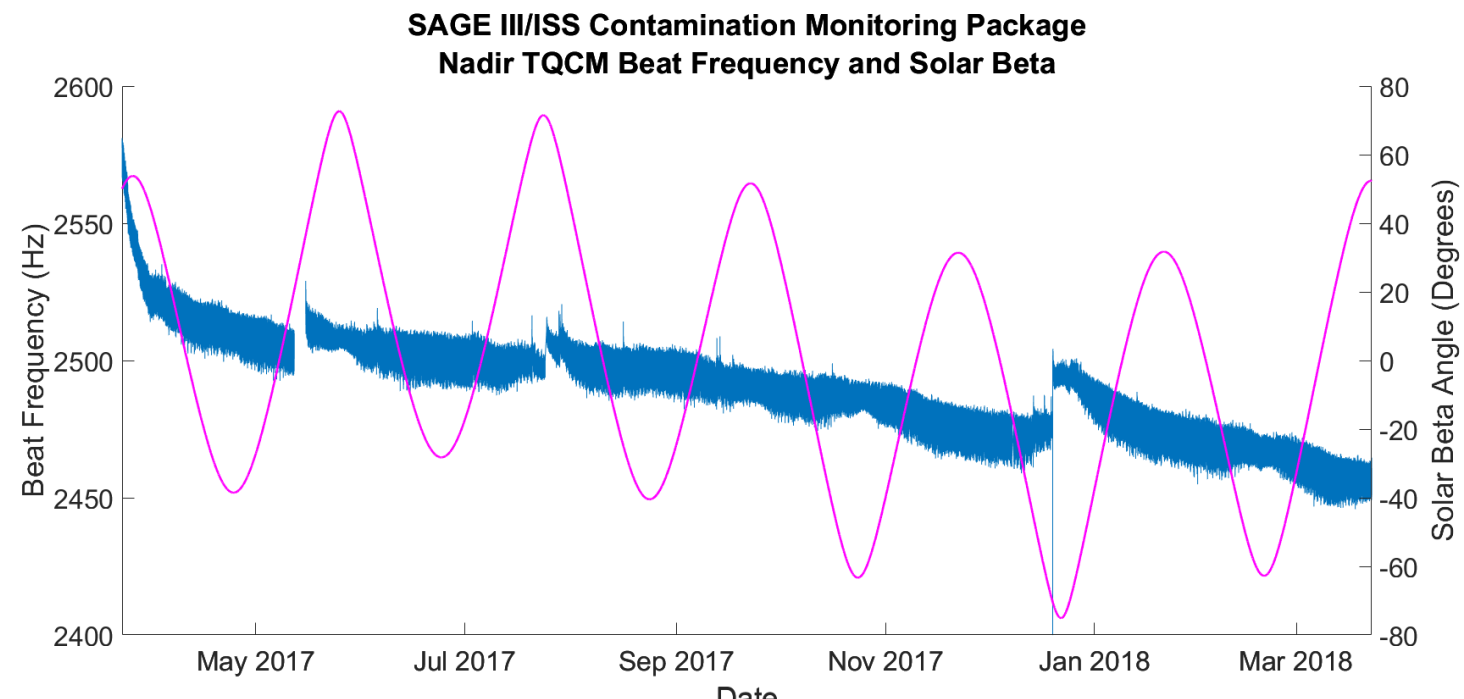

Date

Node 2 Focused Beat Frequency — Solar Beta Angle

Figure 6. SAGE III Nadir facing TQCM beat frequency

SAGE III/ISS Contamination Monitoring Package Wake TQCM Beat Frequency and Solar Beta

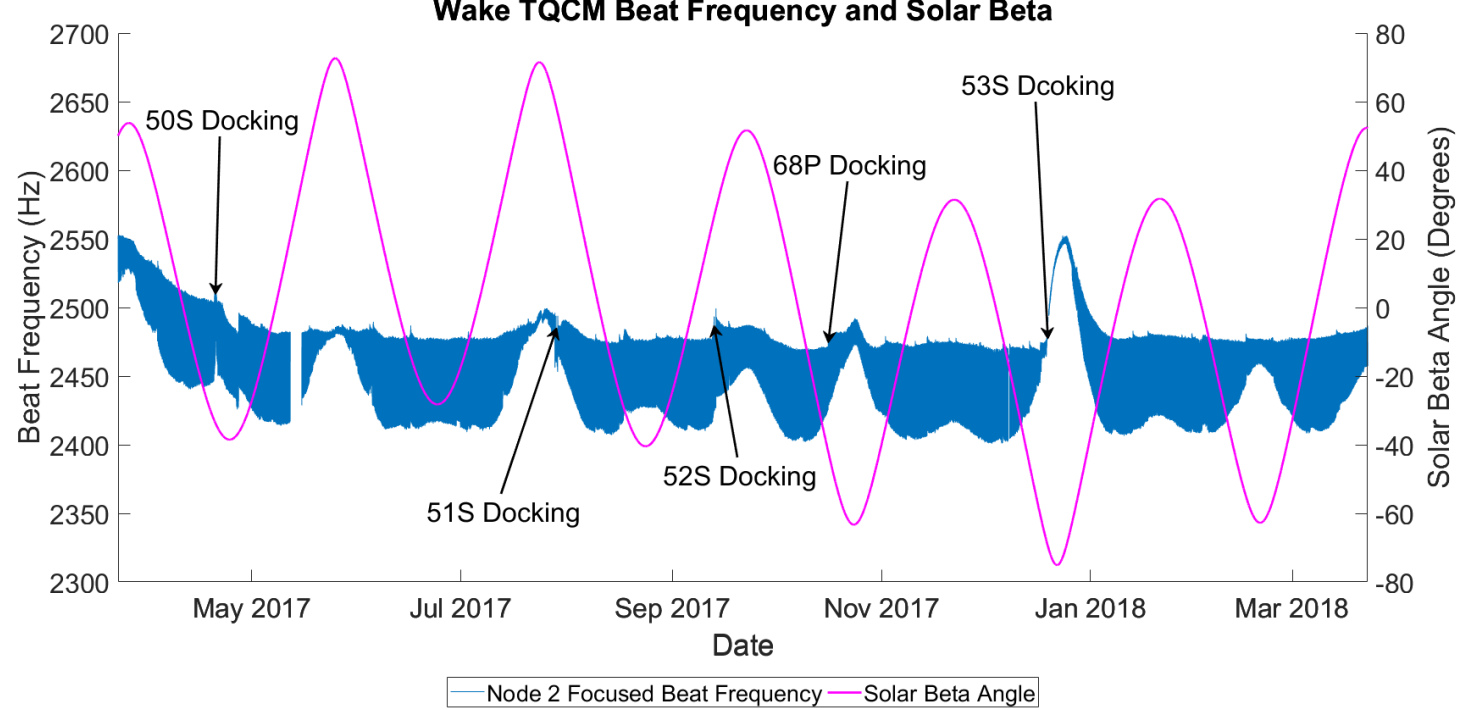

Figure 7. SAGE III Wake facing TQCM beat frequency

The PMM facing TQCM has a field of view directly port of the payload centered on the Permanent Multipurpose Module. This includes the majority of the stations structure as well the berthing locations of Dragon, Cygnus and docking locations for Soyuz and Progress vehicles Figure8 is beat frequency of this TQCM. The beat frequency has visible in it the permanent adsorption associated with Dragon vehicles . As well as the temporary adsorption characteristic of Soyuz and Progress vehicles

The MLM facing TQCM has an inboard field of view from SAGE IIIs site on ELC 4. This field of view 


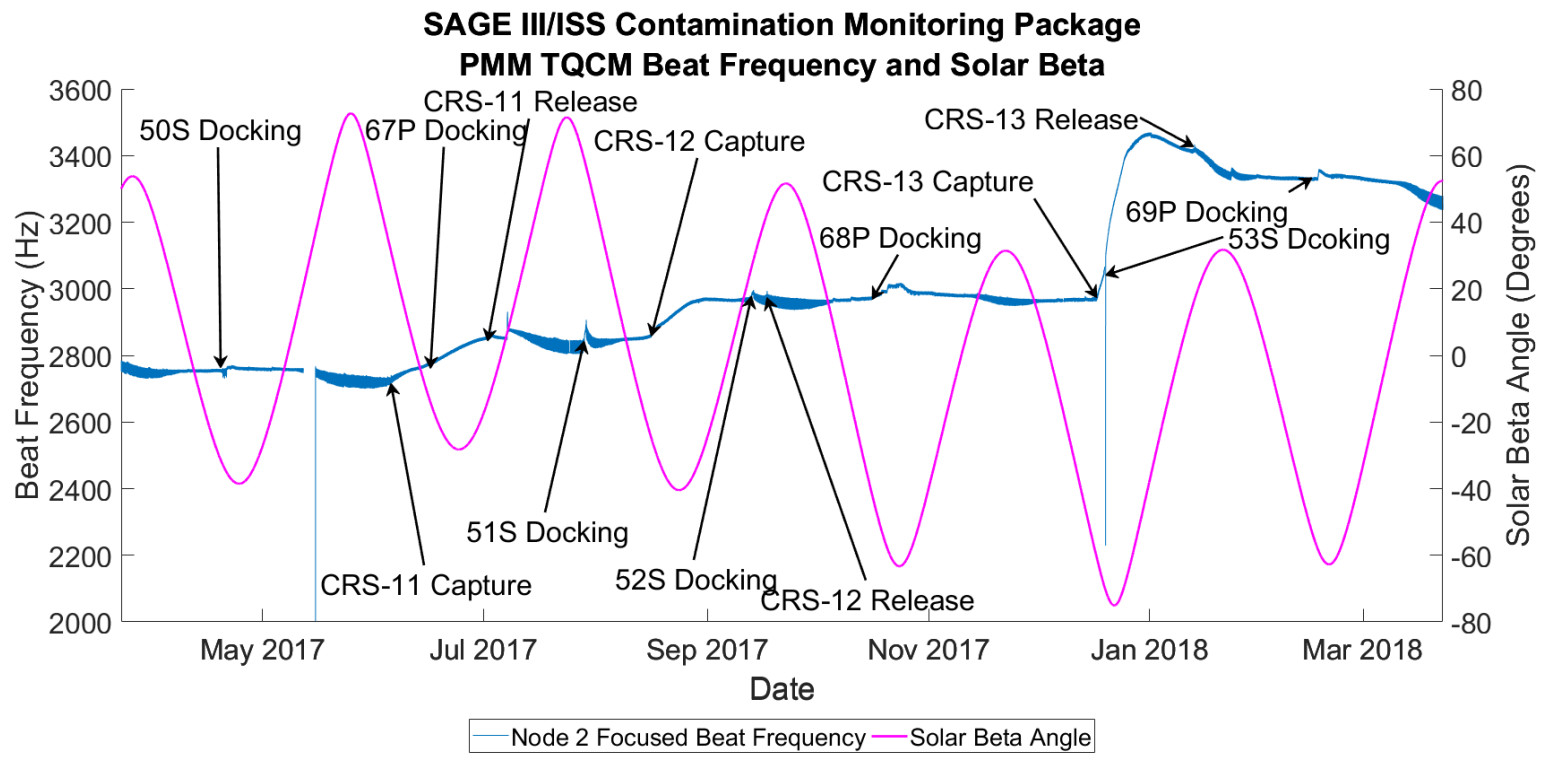

Figure 8. SAGE III PMM facing TQCM beat frequency

contains docking locations for Soyuz and Progress vehicles and is centered on the site that the Multipurpose Laboratory module will be added to station. Figure 9 is the beat frequncy of the MLM TQCM. This TQCM just has the best observations of just Soyuz and Progress vehicles with their characteristic signal of material absorbing onto the TQCM then desorbing off of the TQCM

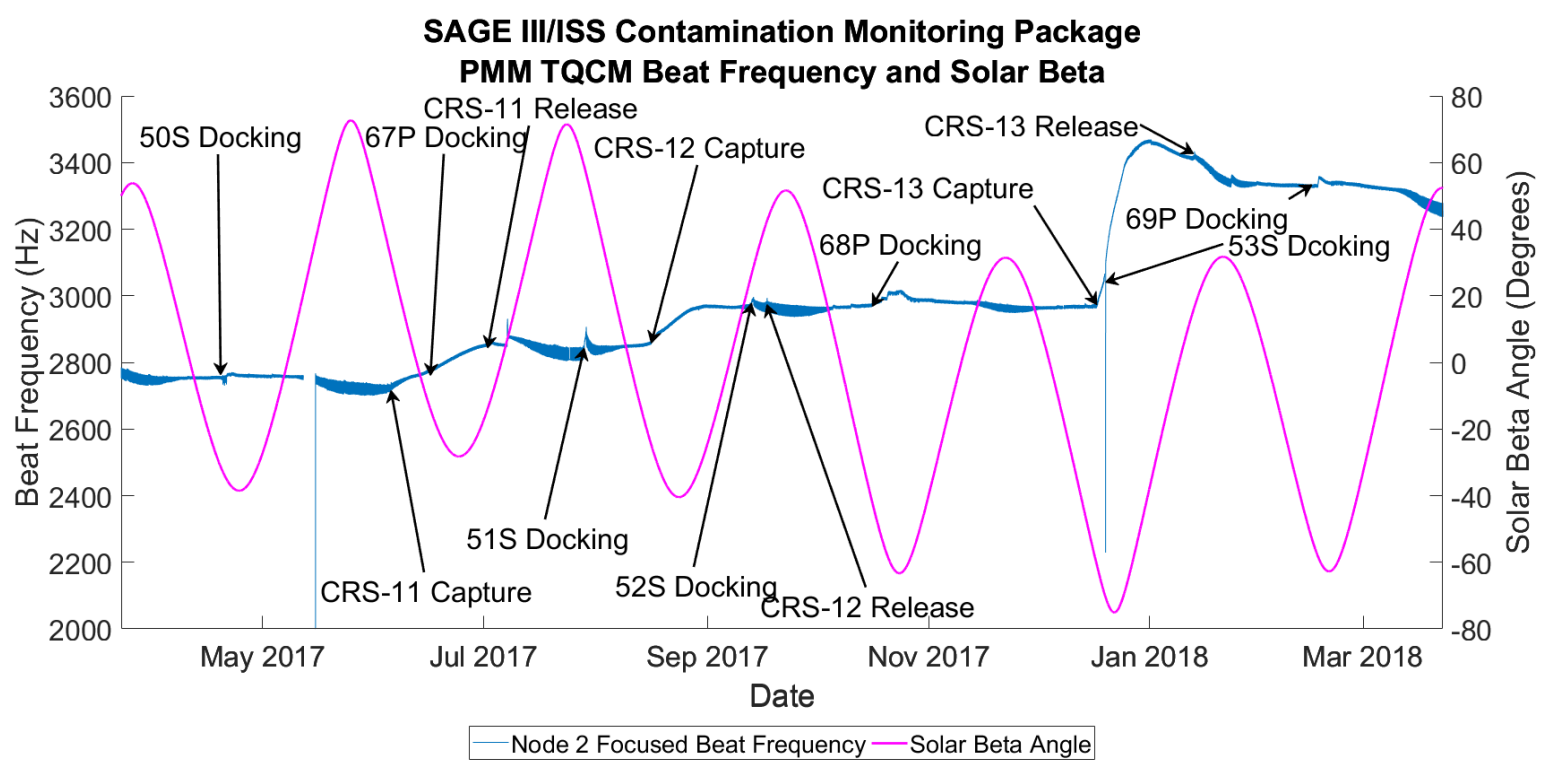

Figure 9. SAGE III MLM facing TQCM beat frequency

Though sunwise TQCMs were used these sensors are still affected by incident solar light causing an orbital 
dependent noise in the beat frequency. It was observed that this noise grew and shrunk in magnitude dependent on the solar beta angle. Solar beta affects the amount of illumination the sensor saw leading to a noisier period when the sensor saw more illumination and a quieter period when the sensor was shadowed by station architecture.

Adsorption estimates for the SAGE III Instrument sensitive surfaces as well as totals for each TQCM are listed in Table 1. Adsorption on the sensitive surface were estimated by using a script to create a virtual CMP on top of the scan head. This virtual CMP rotates with the scan head so that the sensors are always pointing in the same direction relative to the scan head. The beat frequency signal of the virtual CMP is generated by combining the changes in beat frequency signals of the other real TQCMS that it shared a field of view with for each time step. The virtual beat frequency was the result of taking the average change in frequencies of all TQCMs with a field of view around the virtual sensor for each time step. The resulting delta value was used as the delta value for the virtual beat frequency for the time step in which it occurred. The adsorption totals for each TQCM were the differences between the last orbital max frequency value and the first orbital max frequency value for the first year on orbit.

Table 1. Adsorption estimates for SAGE III sensitive surfaces and adsorption totals for SAGE III TQCMs .

\begin{tabular}{|c|c|c|c|}
\hline Adsorption Surface & Total Beat Frequency Change & Total Adsorption $\AA$ & Total Adsorption $\frac{\mathrm{ng}}{\mathrm{cm}^{2}}$ \\
\hline Contamination Window & 137 & 27.41 & 269.3 \\
\hline Scan Head Radiator & 881.3 & 176.26 & 1731.8 \\
\hline Starboard TQCM & 117 & 23.4 & 229.9 \\
\hline Ram TQCM & 86.2 & 17.2 & 169.3 \\
\hline Node 2 Focused TQCM & 1631.5 & 326.3 & 3205.9 \\
\hline Node 2 TQCM & 1642.6 & 328.5 & 3227.6 \\
\hline Nadir TQCM & -117.2 & -23.4 & -230.3 \\
\hline Wake TQCM & -65.4 & -13.1 & -128.5 \\
\hline PMM TQCM & 492.8 & 98.6 & 968.3 \\
\hline MLM TQCM & 79.5 & 15.9 & 156.2 \\
\hline
\end{tabular}

Optical transmission through the contamination window decreases during periods of increased material adsorption. Figure 10 is the contamination window transmission for light wavelengths critical to SAGE III science. The most notable events have occurred during the visits of of CRS vehicles; where elevated levels of material adsorption have been noticed on the Node 2 TQCMs. The use of the Node 2 TQCMs in determining possible affects from contamination on window transmission analysis is due to its optimal location well within the viewing pane of the CRS berth location. Following the CRS-11, CRS-12, CRS-13, and CRS-14 unberthings, the decreased optical transmission of the contamination window clears-up approximately within one month. With SAGE III/ISS just recently surpassing one-year on-orbit, it should be noted that the contamination window transmission change has been minimal, and the impact to SAGE III/ISS science collection is negligible. Currently the internal instrument system response has not changed. Indicating that the observed material adsorption has not degraded the instruments optics and currently SAGE III/ISS has detected no significant long-term optical degradation of the contamination window

\section{CONCLUSION}

Through their first year on orbit the SAGE III CMPs and their TQCMs have been instrumental in detecting periods of increased contamination too the payload. The Node 2 facing TQCMs were pivotal in detecting a large amount of material being out gassed from CRS vehicles. As a result SAGE III was able to close its contamination window in a timely fashion during the visit of CRS-11 when elevated material adsorption levels were first detected and now keeps its contamination window closed during Dragon capsule visits. This has played a large part in 


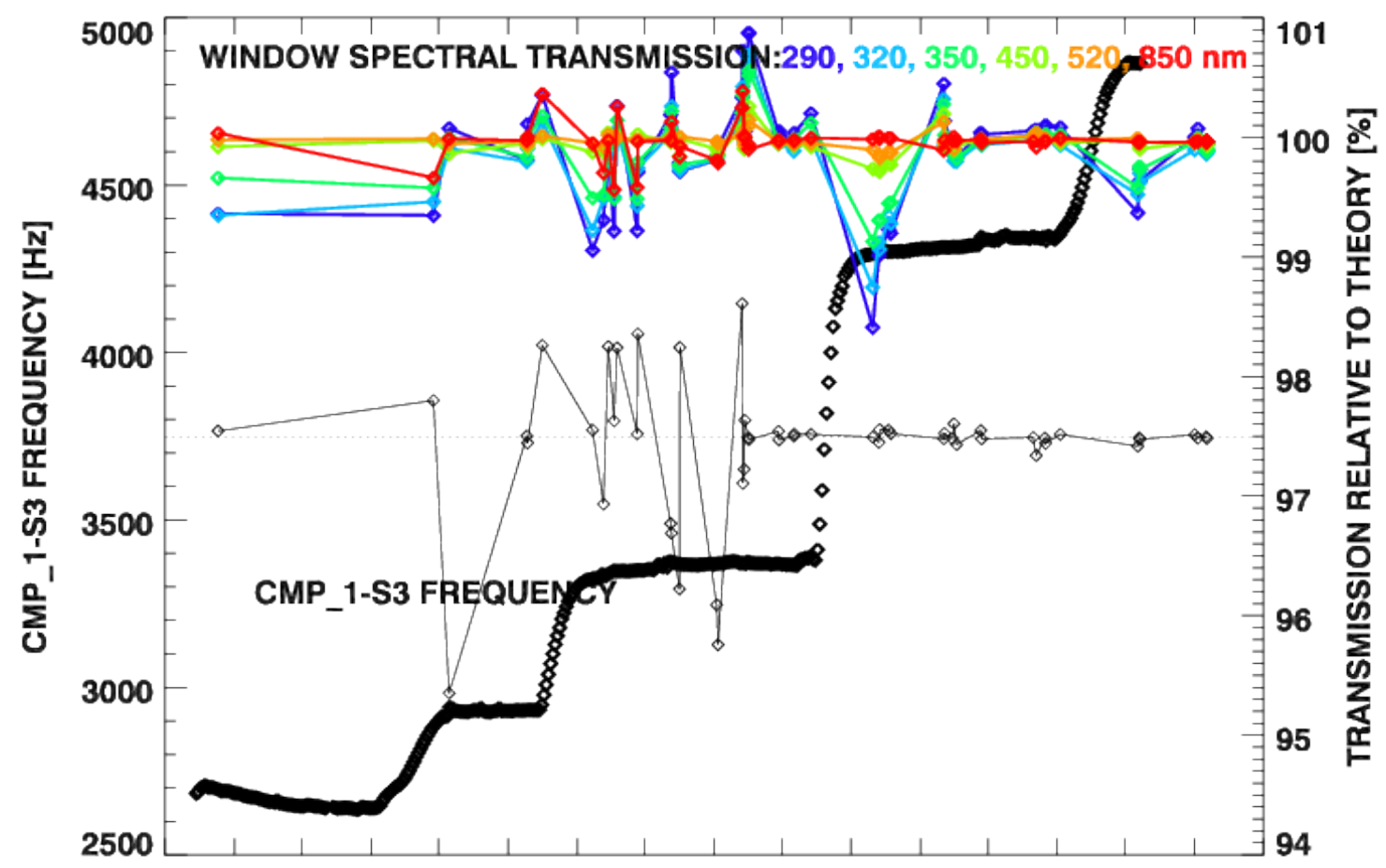

Mar Apr May Jun Jul Aug Sep Oct Nov Dec Jan Feb Mar Apr May Jun Jul

2018

Figure 10. Optical transmission through the contamination door for wavelengths of 290, 320, 350, 450, 520, 850 nm. The beat frequency for the Node 2 TQCM has been included for ease of correlation of drops in window transmission to material adsorption

preventing degradation to the optics train. Due to the contamination control efforts prior to launch and knowing when to inhibit the contamination window while on orbit, SAGE III/ISS has seen no significant degradation in any of the critical wavelengths for the SAGE III science product. The SAGE III TQCMs have also been able to observe characteristic adsorption signals for the most common vehicles to visit station. Soyuz and Progress vehicles will result in a short period of increased material adsorption on the TQCMs the amount of which correlates to the solar beta angle, this material then desorbs within a month after material adsorption ends. The characteristic signal observed for Dragon vehicles is that of elevated material adsorption with the rate correlated again to how negative the solar beta angle is. Unlike Soyuz and Progress the material adsorbed from Dragon vehicles does not desorb even when the TECs control the TQCM temperature to $80^{\circ} \mathrm{C}$. No meaningful material adsorption has been observed during the visit of Cygnus vehicles. Analysis of light transmission through the contamination window has revealed that during the periods that the window is kept closed due to elevated rates of adsorption on the TQCMs a decrease in signal through the contamination window was observed. However after the rate of adsorption has returned to normal with in a month the transmission through the window has returned to normal levels. 


\section{ACKNOWLEDGMENTS}

The SAGE III Project would like to acknowledge and thank Mr. Carl Maag of T\&M Engineering for his outstanding vision and contributions that made the SAGE III Contamination Monitoring Package possible. Carl is an internationally recognized expert in contamination control, planetary protection, material and processes, and space environment and effects and was the contamination control and materials and process lead during SAGE III development and commissioning.

Carl has previous experience as a principal investigator, co-investigator, and consultant for spaceflight payloads investigating space environmental effects and astrophysics including work with sixteen NASA Space Shuttle payloads and leadership of an ESA sponsored payload assessing the contamination environment/micrometeoroid environment of the Russian MIR Space Station. Carls outstanding background made him the right person to lead development of the CMP concept, provide essential and critical design advise, and devise a measurement strategy that has made the CMP a useful tool supporting SAGE III on-orbit operations while expanding our knowledge of the space environment. Thank you Carl the SAGE III CMPs are working great and are providing data to help all of us advance the state of the art well done!

The SAGE III Project would like to acknowledge and thank the Boeing ISS Space Environments team for their support in assessing the impacts of Dragon contamination as well as reviewing this paper.

\section{REFERENCES}

[1] Lemuel E. Mauldin, Rashid Salikhov, S. H. A. G. V. D. C. G. P. J. C., "Meteor-3m(1)/stratospheric aerosol and gas experiment iii (sage iii) jointly sponsored by the national aeronautics and space administration and the russian space agency," in [Proc.SPIE], 3501 (1998).

[2] Michael Cisewski, Joseph Zawodny, J. G. R. E. N. T. O. R.-A. D. C. S. H., "The stratospheric aerosol and gas experiment (sage iii) on the international space station (iss) mission," in [Proc.SPIE], 9241 (2014).

[3] QCM Research, 41831 Mcalby Ct \# C, Murrieta, CA 92562, MK 24 Thermoelectric Quartz Crystal Microbalance TQCM Sensor Operating Manual (July 2008).

[4] Wood, B. E., Hall, D., Lesho, J., Uy, O., Dyer, J., and Bertrand, W., "Msx satellite flight measurments of contaminant deposition on a cqcm and on tqcms," in [AIAA, Aerospace Sciences Meeting $\&$ Exhibits], (1997). 